\title{
Local Chain Dynamics of Poly(cis-1,4-isoprene) in Dilute Solutions Studied by the Fluorescence Depolarization Method
}

\author{
Keiko Ono, Kazuhiko UedA, and Masahide YамAмOTo* \\ Division of Polymer Chemistry, Graduate School of Engineering, Kyoto University, \\ Sakyo-ku, Kyoto 606, Japan
}

(Received May 9, 1994)

\begin{abstract}
Local segmental motions of poly(cis-1,4-isoprene) (cis-PI) labeled with anthracene were examined by the method of fluorescence depolarization using 1,4-dioxane, $n$-dodecane, cyclohexane, benzene, ethylbenzene, and $o$-xylene as the solvents with a viscosity of less than $2 \mathrm{cP}$. The anisotropy ratio was measured in these dilute solutions, and the mean relaxation time, $T_{\mathrm{m}}$, for the chain local motion was obtained. The activation energy, $E^{*}$, was obtained by using the theory of Kramers' diffusion limit. The reduced relaxation time, $T_{\mathrm{m}} / \eta$, for cis-PI was much shorter than that for polystyrene and was independent of the local segment density differing from that of polystyrene. The dependence of activation energy, $E^{*}$, on the solvent was very weak, compared to that of polystyrene. These findings could be interpreted in terms of the dynamic flexibility of the polymer chain.
\end{abstract}

KEY WORDS Poly(cis-1,4-isoprene)/Fluorescence Depolarization Method / Segment Density / Relaxation Time / Activation Energy / Freely Rotating Chain /

Polymer chains have a wide spectrum of relaxation modes. One of the relaxation mode is a cooperative global motion, so-called normal mode. ${ }^{1-3}$ Another is the local segmental motion, ${ }^{4-6}$ which is considered as the conformational transition in a few repeated units, and its time scale is within a few nano to sub-nano seconds.

Recently, the local motions of polymer chains have been studied by various methods, ${ }^{13} \mathrm{C}$ NMR, ${ }^{7-9}$ dynamic light scattering, ${ }^{10,11}$ $\mathrm{ESR},{ }^{12}$ dielectric relaxation, ${ }^{1,13,14}$ and fluorescence depolarization. ${ }^{15-17}$ We have examined the polymeric local motion by the fluorescence depolarization method. ${ }^{17,18} \mathrm{~A}$ fluorescent anthracene probe was introduced in a chain. As the probe is linked to the polymer chain covalently, the autocorrelation function of a polymer chain can be directly observed by measuring the fluorescence anisotropy ratio without the hypothesis of any models. This is an advantage of the method of fluorescence depolarization.

Poly(cis-1,4-isoprene) (cis-PI) is well-known as a natural rubber. The glass transition temperature, $T_{\mathrm{g}}$, is $c a .-70^{\circ} \mathrm{C}$, and PI has the rubber state at room temperature. As PI has dipoles parallel along the chain, Stockmayer et al., ${ }^{1,2}$ Adachi et al., ${ }^{19-21}$ and others ${ }^{22}$ have examined the entanglement dynamics by the dielectric relaxation method. In a PI dilute solution system, the local segmental dynamics have been studied by Monnerie et $a .^{23}$ and Ediger et al. ${ }^{7,24,25}$ Monnerie et al. examined the local motion of a PI labeled with anthracene in the middle of the chain by the method of fluorescence depolarization. They discussed the decay curve of the anisotropy ratio on the basis of various models with an orientational autocorrelation function. Ediger et al. examined the dependence of the PI local chain dynamics on the molecular weight and the 
anomalous activation energy of PI in a range of highly viscous solvents by the fluorescence depolarization technique. Here, the cis content of the anthracene-labeled PI samples was low, $39-54 \%$. They also studied the activation energy for cis-PI in highly viscous solvents by ${ }^{13} \mathrm{C}$ NMR.

We report the local segmental dynamics of cis-PI in various dilute solutions studied by the fluorescence depolarization technique. Previously, Adolf et al. ${ }^{25}$ examined the local motion of cis-PI by the same method of fluorescence depolarization, but the cis content of their PI sample was small, $54 \%$. Here, we synthesized anthracene-labeled cis-PI with a higher cis content, $76 \%$. We discuss the effects of solvents on the activation energy and the relaxation time in a solvent of low viscosity.

\section{EXPERIMENTAL}

\section{Materials}

The anthracene-labeled cis-PI as a polymer sample was prepared by anionic polymerization; the living ends were coupled by 9,10 bis(bromomethyl)anthracene (Figure 1).

The anionic polymerization of isoprene monomer was initiated by sec-butyllithium in benzene at room temperature. The polymerization reaction was allowed to proceed at room temperature. When the whole monomer was exhausted, 9,10-bis(bromomethyl)anthracene which had been dissolved in tetrahydrofuran (THF), was added to the reaction vessel, and the solution was stirred for 24 hours. The polymer was purified by reprecipitation from benzene in methanol. Furthermore, we fractionated the part of cis-PI labeled in the middle of a main chain by GPC. The molecular weight and the content of stereoisomers were measured by GPC and ${ }^{13} \mathrm{C}$ NMR, respectively

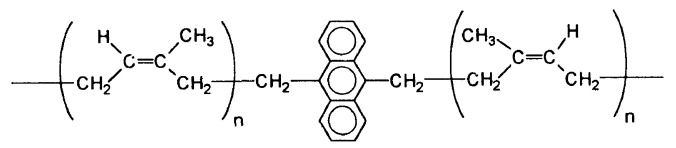

Figure 1. Cis-PI sample labeled with anthracene.
(Table I).

We used 6 kinds of solvents, i.e., 1,4-dioxane (Nacalai Tesque, Spectrophotometric Grade, (S)), $n$-dodecane (Nacalai Tesque, Guaranteed Reagent, (G)), benzene (Nacalai Tesque, S), ethylbenzene (Waken, S), cyclohexane (Nacalai Tesque, S), and $o$-xylene (Nacalai Tesque, G). Table II shows that their viscosities ${ }^{26}$ and values are relatively low, less than $2 \mathrm{cP}$ at room temperature. They were used as received without distillation. We prepared these cis-PI solutions in quartz cells at a concentration of less than $0.2 \mathrm{wt} \%$. The samples were degassed by freeze-to-pump-thawing.

\section{Intrinsic Viscosity}

The intrinsic viscosity for each solution was measured by capillary viscometry. We used a polyisoprene standard sample (Scientific Polymer Products, Inc. CAT\#706) as a polymer sample, whose characterization is; $M_{w}=80.3 \mathrm{k}$, $M_{n}=59.1 \mathrm{k}, M_{w} / M_{n}=1.36$, cis $-1,4$ content $=$ $71 \%$. We selected this sample because it has almost the same cis-content as our labeled cis-PI. We evaluated the chain expansion factor, $\bar{a}_{\eta}^{3}$, which is the ratio of the intrinsic viscosity of a sample solution to that in a $\theta$ solvent at $\theta$ temperature. In this study, dioxane at $34^{\circ} \mathrm{C}$ was regarded as a $\theta$ condition for cis-PI. ${ }^{27,28}$

Table I. Characterization of cis-PI sample

\begin{tabular}{cccccc}
\hline $\begin{array}{c}M_{n} \\
\times 10^{-4}\end{array}$ & $\begin{array}{c}M_{w} \\
\times 10^{-4}\end{array}$ & $M_{w} / M_{n}$ & $\begin{array}{c}\text { cis-1,4 } \\
\%\end{array}$ & $\begin{array}{c}\text { trans-1,4 } \\
\%\end{array}$ & $\begin{array}{c}\text { Vinyl } \\
\%\end{array}$ \\
\hline 13.1 & 14.5 & 1.10 & 76 & 19 & 5 \\
\hline
\end{tabular}

Table II. Solvent viscosity at $20^{\circ} \mathrm{C}$, and chain expansion factor, $\bar{a}_{\eta}^{3}$, for PI at $34^{\circ} \mathrm{C}$

\begin{tabular}{lll}
\hline Solvent & $\eta / \mathrm{cP}$ & $\overline{a_{\eta}{ }^{3}}$ \\
\hline Dioxane & 1.31 & 1.00 \\
$n$-Dodecane & 1.47 & 1.27 \\
Ethylbenzene & 0.68 & 1.76 \\
Benzene & 0.65 & 1.77 \\
Cyclohexane & 0.95 & 1.83 \\
$o$-Xylene & 0.83 & 1.89 \\
\hline
\end{tabular}


Time-Resolved Fluorescence Depolarization Method

We measured the time-resolved fluorescence anisotropy ratio of a polymer chain by a single-photon counting system, as described in detail previously. ${ }^{29} \mathrm{~A}$ diode laser (Hamamatsu Photonics PLP-01, $1 \mathrm{MHz}$ ) was used as an excitating light source. The excitation light was plane-polarized in the vertical direction and the wavelength was $411 \mathrm{~nm}$. We used a microchannel plate photomultiplier tube (Hamamatsu Photonics) as a detector. The value of FWHM in this system was $c a .300$ ps. Cut-off filters, Y-44 and V-42, and a polarizer were put in front of the detector, and the emission intensities in the vertical direction $\left(I_{\mathrm{VV}}(t)\right)$ and horizontal direction $\left(I_{\mathrm{VH}}(t)\right)$ were collected as the function of time, $t$. The total fluorescence intensity decay, $I^{\text {obsd }}(t)$, was defined as

$$
I^{\text {obsd }}(t)=I_{\mathrm{VV}}(t)+2 G \cdot I_{\mathrm{VH}}(t)
$$

where $G$ is the instrumental specific parameter, so-called $G$-factor. Here, we took $G=1.0094$. $I^{\text {calcd }}(t)$ was evaluated by convolution of $I(t)$ by the instrumental function, $P(t)$.

$$
I^{\text {calcd }}\left(t_{\mathrm{i}}\right)=\int_{0}^{t_{\mathrm{i}}} P(T) I\left(t_{\mathrm{i}}-T\right) \mathrm{d} T
$$

where we used eq 3 as the function for $I(t)$.

$$
I(t)=a_{1} \exp \left(-t / \tau_{1}\right)+a_{2} \exp \left(-t / \tau_{2}\right)
$$

The parameters of $I(t)$ could be estimated by fitting $I^{\text {calcd }}(t)$ to $I^{\text {obsd }}(t)$ by the method of nonlinear-least-squares. ${ }^{30}$

\section{Analysis of $r(t)$}

The time-resolved anisotropy ratio was defined as

$$
r^{\text {obsd }}(t)=\frac{\left(I_{\mathrm{VV}}(t)-G \cdot I_{\mathrm{VH}}(t)\right)}{\left(I_{\mathrm{VV}}(t)+2 G \cdot I_{\mathrm{VH}}(t)\right)}
$$

The anisotropy ratio, $r(t)$, is related to the orientational autocorrelation function, $\Phi(t)$, as

$$
r(t)=r_{0} \cdot \Phi(t)
$$

where $r_{0}$ is the initial anistropy ratio. We used an empirical equation, eq 6 , as $\Phi(t)$.

$\Phi(t)=x \exp \left(-t / T_{1}\right)+(1-x) \exp \left(-t / T_{2}\right)$

Equation 6 shows a very good agreement with the anisotropy decay data in a wide viscosity range. $r^{\text {calcd }}(t)$ was also evaluated by convolution as

$r^{\text {calcd }}(t)=\frac{r_{0} \int_{0}^{t_{\mathrm{i}}} P(T) \Phi\left(t_{\mathrm{i}}-T\right) I\left(t_{\mathrm{i}}-T\right) \mathrm{d} T}{\int_{0}^{t_{\mathrm{i}}} P(T) I\left(t_{\mathrm{i}}-T\right) \mathrm{d} T}$

$r^{\text {calcd }}(t)$ was also fitted to $r^{\text {obsd }}(t)$ by the method of nonlinear-least-squares with weighting factor propagated Poisson error ${ }^{30}$ and $x, T_{1}, T_{2}$, and $r_{0}$ were determined as the best-fit parameters. The mean relaxation time was defined as

$$
\begin{aligned}
T_{\mathrm{m}} & =\int_{0}^{\infty} \Phi(t) \mathrm{d} t \\
& =x T_{1}+(1-x) T_{2}
\end{aligned}
$$

Since the function of eq 6 is empirical, the physical meaning of $T_{1}$ and $T_{2}$ is not definitive. The parameters, $T_{1}$ and $T_{2}$, may reflect two kinds of the representative motional modes, e.g., an isolated transition and the cooperative counter-rotation in the Hall and Helfand model. ${ }^{4,31}$

\section{Evaluation of an Activation Energy}

We evaluated the activation energy for the conformational transition of a polymer chain by the theory of Kramers' diffusion limit. ${ }^{32}$ The mean relaxation time, $T_{\mathrm{m}}$, is represented by

$$
T_{\mathrm{m}}=A \eta \exp \left(E^{*} / R T\right)
$$

where $\eta$ is solvent viscosity, $E^{*}$ is apparent activation energy, $R$ is the gas constant and $T$ is the absolute temperature. When a solvent viscosity is described by the Arrhenius type equation with an activation energy, $E_{\eta}$,

$$
\eta=\eta_{0} \exp \left(E_{\eta} / R T\right)
$$


eq 9 can be written as eq 11 .

$$
T_{\mathrm{m}}=A \eta_{0} \exp \left\{\left(E^{*}+E_{\eta}\right) / R T\right\}
$$

When we make the Arrhenius plots of $\ln \left(T_{\mathrm{m}}\right)$ against $1 / T$, we can obtain the value of $\left(E^{*}+E_{\eta}\right)$ from the slope of a straight line. The value of $E^{*}$ can be obtained by subtracting $E_{\eta}$ from the value of the slope.

\section{RESULTS AND DISCUSSION}

\section{The Relaxation Time}

We obtained the value of the chain expansion factor, ${\overline{a_{\eta}}}^{3}$, in each solution by an intrinsic viscosity measurement. Table II shows the value of ${\overline{a_{\eta}}}^{3}$ in each solution. 1,4-Dioxane is a $\theta$ solvent for cis-PI, and the solvent becomes better in the order of $n$-dodecane, ethylbenzene, benzene, cyclohexane, and $o$-xylene.

Table II and Table III show the relationship between the reduced relaxation time, $T_{\mathrm{m}} / \eta$, and the chain expansion. The value of ${\overline{a_{\eta}}}^{3}$ corresponds to the reciprocal of chain segmental density, and the dependence of the reduced relaxation time of cis-PI on the segment density was not appreciable. In our previous paper, ${ }^{33}$ the reduced relaxation time of polystyrene (PS) became shorter, as the segment density became smaller. The $T_{\mathrm{m}} / \eta$ of PS in a $\theta$ solvent, cyclohexane, was $5.4 \mathrm{~ns} \mathrm{cP}^{-1}$, and the $T_{\mathrm{m}} / \eta$ in good solvents, benzene and ethylbenzene, was $2.7 \mathrm{~ns} \mathrm{cP}^{-1}$ and $2.9 \mathrm{~ns} \mathrm{cP}^{-1}$ at $34^{\circ} \mathrm{C}$, respectively. The value of $T_{\mathrm{m}} / \eta$ in a $\theta$ solvent is about two times than that in good solvents. As for poly $(\alpha$-methylstyrene) (P $\alpha \mathrm{MS})$

Table III. Reduced relaxation time, $T_{\mathrm{m}} / \eta$, for PI solutions at $34^{\circ} \mathrm{C}$

\begin{tabular}{lc}
\hline Solvent & $T_{\mathrm{m}} / \eta / \mathrm{ns} \mathrm{cP}^{-1}$ \\
\hline Dioxane & 1.33 \\
$n$-Dodecane & 1.29 \\
Ethylbenzene & 1.21 \\
Benzene & 1.28 \\
Cyclohexane & 1.51 \\
$o$-Xylene & 1.32 \\
\hline
\end{tabular}

and poly( $p$-methylstyrene) (PpMS), the $T_{\mathrm{m}} / \eta$ similarly became shorter, as the segment density became smaller.

The local motion of PI in a dilute solution was faster than those of PS, $\mathrm{P} \alpha \mathrm{MS}$, and $\mathrm{P} p \mathrm{MS}$. For example, in a $\theta$ solvent, $T_{\mathrm{m}} / \eta$ of PS was $5.4 \mathrm{~ns} \mathrm{cP}^{-1}$ and that of PI was $1.3 \mathrm{nscP}^{-1}$ at $34^{\circ} \mathrm{C}$. Here, we will consider the chain local motion from the standpoint of the degree of the freedom for a chain rotation. The meansquare end-to-end distance in the unperturbed chain is represented by $\left\langle R_{\mathrm{o}}^{2}\right\rangle$, and that in the unperturbed chain calculated by hypothesis of freely rotating chain is represented by $\left\langle R_{\text {of }}^{2}\right\rangle$. The ratio, $\left(\left\langle R_{\mathrm{o}}^{2}\right\rangle /\left\langle R_{\text {of }}^{2}\right\rangle\right)^{1 / 2}$, is specific for the kind of polymer, and indicates the degree of freedom of rotation around the bond, in other words, the degree of the steric hindrance for rotation of each polymer chain in comparison with the freely rotating chain. When $\left(\left\langle R_{\mathrm{o}}^{2}\right\rangle \mid\right.$ $\left.\left\langle R_{\text {of }}^{2}\right\rangle\right)^{1 / 2}$ becomes near unity, the chain approaches a condition of a freely rotating chain. According to ref 34 , the value of $\left(\left\langle R_{\mathrm{o}}^{2}\right\rangle /\left\langle R_{\mathrm{of}}^{2}\right\rangle\right)^{1 / 2}$ for PS is 2.44 at ca. $25^{\circ} \mathrm{C}$ and 2.35 at $70^{\circ} \mathrm{C}$, and that for cis-PI (natural rubber) is 1.71 in the range of $0-60^{\circ} \mathrm{C}$. The PS chain has a bulky phenyl group in every other carbon in the main chain. Therefore, the steric hindrance for the rotation around the main chain is large. However, the cis-PI chain has a double bond and does not have a bulky side chain. Such a molecular structure makes a chain freely rotate in some degree. The fact that the degree of freedom for the rotation of cis-PI chain is larger than that of the PS chain, affects clearly the relaxation of a local chain motion. This shows that the fast relaxation of cis-PI corresponds to the degree of freedom of internal rotation in the molecular structure.

Furthermore, the fact that the reduced relaxation time of cis-PI was almost independent of the chain expansion means that the long range interaction does not affect the local motion of cis-PI. The long range interaction indicates the intramolecular interaction caused by the excluded volume effect, 
and the short range interaction indicates the intramolecular interaction caused by the conformation of a few bonds or by a rotation around a bond. The local conformational transition of cis-PI caused by a few repeated units is little affected by a long range interaction since the rotation of cis-PI chain is rather free compared with that of PS. By contrast, in the chain with a large steric hindrance for the chain rotation, the local motion strongly depends on the chain expansion.

Next, we examined whether the friction between polymer segments and solvent molecules is proportional to solvent viscosity. Adolf et $a l .{ }^{25}$ reported for PI solutions that in high viscosity solvents, the theory of Kramers' diffusion limit cannot be used to evaluate the activation energy, i.e., the friction between polymer segments and solvent molecules is not proportional to solvent viscosity. They plotted $\ln \left(T_{\mathrm{m}}\right)$ against $\ln (\eta)$, and obtained a slope of 0.41 . When the friction is proportional to the solvent viscosity, the value of the slope should be unity. In this study, we used the solvents with relatively low viscosities of less than $2 \mathrm{cP}$ at $20^{\circ} \mathrm{C}$. Figure 2 shows the relationship between $T_{\mathrm{m}}$ and the solvent viscosity at $34^{\circ} \mathrm{C}$ for PI solutions; the slope of $\ln \left(T_{\mathrm{m}}\right)$ against $\ln (\eta)$ is unity. The activation energy can be

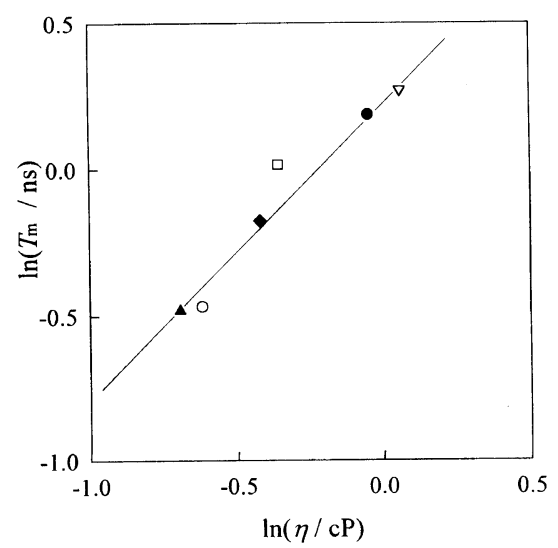

Figure 2. The plots of $T_{\mathrm{m}}$ for cis-PI against the solvent viscosity at $34^{\circ} \mathrm{C} . \nabla, n$-dodecane; $\bigcirc, 1,4$-dioxane; $\square$, cyclohexane; $\bullet, o$-xylene; $\bigcirc$, ethylbenzene; $\boldsymbol{\Delta}$, benzene. evaluated by the theory of Kramers' diffusion limit in the solvents below $2 \mathrm{cP}$. Adolf et al. ${ }^{25}$ reported the same finding for the solvents of viscosity below $2 \mathrm{cP}$ (at $46^{\circ} \mathrm{C}$ ).

In our previous paper, ${ }^{29}$ for the dynamics of PS in a highly viscous solvent, the slope of $\ln \left(T_{\mathrm{m}}\right)$ against $\ln (\eta)$ was less than unity. Such a result was anomalous, and it was considered that the solvent of high viscosity makes a kind of structure and the polymer chain moves in such an ordered solvent.

\section{The Activation Energy for cis-PI}

Figure 3 shows the Arrhenius plots of $\ln \left(T_{\mathrm{m}}\right)$ against $I / T$. The slopes of these straight lines represent the values of $\left(E^{*}+E_{\eta}\right) . E^{*}$ was determined by subtracting $E_{\eta}$ from the value of the slope, and we show the activation energy for a polymer chain, $E^{*}$, in Table IV.

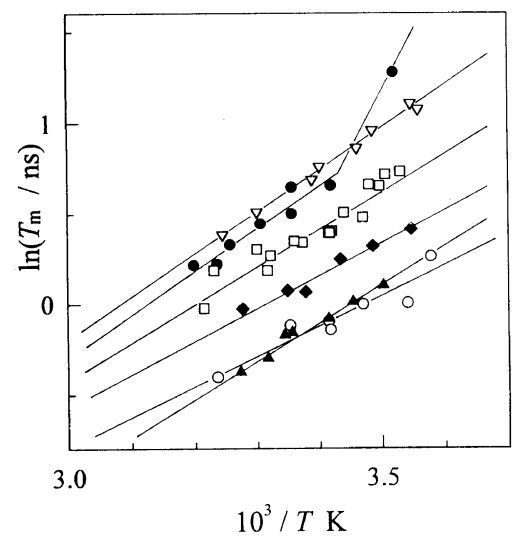

Figure 3. The Arrhenius plots of $\ln \left(T_{\mathrm{m}} / \mathrm{ns}\right)$ against $1 / T$ for each PI solution. Symbols are the same as those in Figure 2.

Table IV. Activation energy for the local motion of cis-PI, $E^{*}$, and that for the solvent viscosity, $E_{\eta}$

\begin{tabular}{lcc}
\hline \multicolumn{1}{r}{ Solvent } & $E^{*} / \mathrm{kcal} \mathrm{mol}^{-1}$ & $E_{\eta} / \mathrm{kcal} \mathrm{mol}^{-1}$ \\
\hline Dioxane & 1.5 & 3.0 \\
$n$-Dodecane & 1.6 & 3.0 \\
Ethylbenzene & 1.2 & 2.2 \\
Benzene & 1.3 & 2.5 \\
Cyclohexane & 1.3 & 2.9 \\
$o$-Xylene & 1.2 & 2.2
\end{tabular}




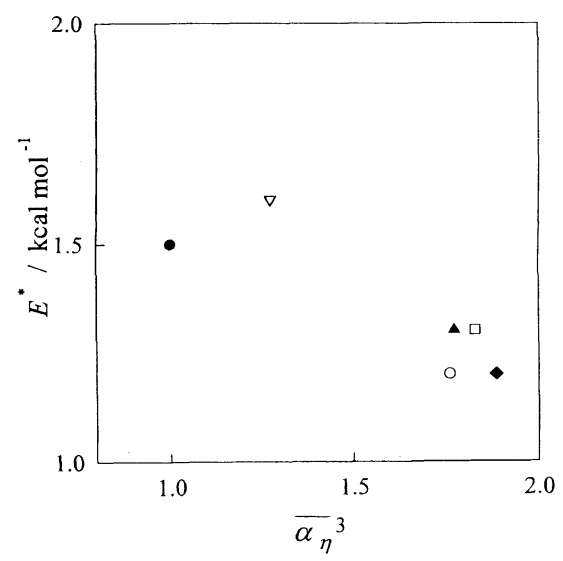

Figure 4. The plots of $E^{*}$ for cis-PI against the chain expansion factor, $\bar{a}_{\eta}^{3}$, at $34^{\circ} \mathrm{C}$. Symbols are the same as those in Figure 2.

1,4-Dioxane is a $\theta$ solvent for cis-PI, and the $\theta$ temperature is $34^{\circ} \mathrm{C}$. The slope of the straight line for dioxane solution inflects at $c a .15^{\circ} \mathrm{C}$ below $\theta$ temperature. We ascribe this point to an intramolecular microaggregation. ${ }^{33}$

Figure 4 shows the relationship between the activation energy and the chain expansion. For PI solutions, the dependence of $E^{*}$ on the chain expansion, was very weak. The value of $E^{*}$ in a $\theta$ solvent was $1.5 \mathrm{kcal} \mathrm{mol}^{-1}$, and the values in good solvents were in the range of $1.2-1.3 \mathrm{kcal} \mathrm{mol}^{-1}$. This weak dependence of the activation energy on the segment density is due to the character of the cis-PI chain, that is, the weak influence of long range interaction due to the small steric hindrance for the rotation around the bonds of cis-PI chain as described in the previous section.

Previously, we reported ${ }^{33}$ the relationship between the activation energy and the segment density in a series of styrene polymers. For PS solutions, $E^{*}$ pronouncedly became lower with the expansion of the polymer chain. The activation energy in a $\theta$ solvent, cyclohexane, was $2.6 \mathrm{kcal} \mathrm{mol}^{-1}$ and those in good solvents, ethylbenzene and toluene, were $1.3 \mathrm{kcal} \mathrm{mol}^{-1}$ and $1.4 \mathrm{kcal} \mathrm{mol}^{-1}$, respectively. $\mathrm{P} \alpha \mathrm{MS}$ and $\mathrm{P} p \mathrm{MS}$ solutions also showed the same tendency; the value of $E^{*}$ in a $\theta$ solvent was higher above $1 \mathrm{kcal} \mathrm{mol}^{-1}$ than those in good solvents. As described in ref 33, the value of an intrinsic viscosity, i.e., the segment density, changes most steeply near the $\theta$ temperature. In poor solvents, $E^{*}$ is large, because the temperature dependence of the polymeric segment density is larger than that in good solvents. This explains why the activation energy for PS becomes smaller as the segment density becomes smaller.

Now, we discuss the effect of stereoregularity on the local motion of polymer chains. Adolf et al. $^{25}$ examined the local motion of PI with a cis-1,4 content of $54 \%$ by the fluorescence depolarization method. They observed the activation energies in solvents in a wide viscosity range, $0.4-35 \mathrm{cP}$ at $45^{\circ} \mathrm{C}$. The activation energy in toluene, cyclohexane, and $n$-dodecane solvents was $1.7-1.8 \mathrm{kcal} \mathrm{mol}^{-1}$. These values are slightly larger than ours, $1.2-1.6 \mathrm{kcal} \mathrm{mol}^{-1}$. We consider that cis-PI with a higher cis-content makes lower activation energy, however, we will need more experiments to determine the relationship between the local motion of PI and its stereoregularity.

Secondly, Glowinkowski et al. ${ }^{7}$ reported the local segmental dynamics of PI with cis-1,4 content $=70-80 \%$ by ${ }^{13} \mathrm{C}$ NMR. They also used the solvents in a wide viscosity range. In the solvents, toluene, chloroform, cyclohexane, and 1,4-dioxane, the activation energies were $1.4-1.7 \mathrm{kcal} \mathrm{mol}^{-1}$, which are in good agreement with those we obtained. This agreement may be cautiously compared since the relaxation times they obtained, $c a \cdot 10^{-10}-10^{-11} \mathrm{~s}$, are in one order of magnitude smaller than those we obtained, $c a \cdot 10^{-9} \mathrm{~s}$. The results obtained by different techniques may reflect different part of a relaxation time distribution.

\section{CONCLUSION}

We measured the local segmental motions of $c i s$-PI labeled with anthracene in the middle of the main chain by the fluorescence 
depolarization technique. The reduced relaxation time, $T_{\mathrm{m}} / \eta$, in various solvents was independent of the segment density, and the dependence of $E^{*}$ on the segment density was very weak. The findings were different from our previous result obtained for PS solutions. These findings may reflect the small steric hindrance for the rotation of cis-PI chain.

Acknowledgments. We thank Dr. Hirokazu Hasegawa of Kyoto University for kind guidance in the preparation of anthracene labeled cis-PI by anionic polymerization.

\section{REFERENCES}

1. W. H. Stockmayer, Pure Appl. Chem., 15, 539 (1967).

2. W. H. Stockmayer and J. J. Burke, Macromolecules, 2, 647 (1969).

3. M. Doi and S. F. Edwards, "The Theory of Polymer Dynamics," Oxford Science Publishers, Oxford, 1986.

4. E. Helfand, J. Chem. Phys., 54, 4651 (1971).

5. E. Helfand, Z. R. Wasserman, and T. A. Weber, Macromolecules, 13, 526 (1980).

6. T. Yoshizaki, M. Fujii, and H. Yamakawa, J. Chem. Phys., 82, 1003 (1985).

7. S. Glowinkowski, D. J. Gisser, and M. D. Ediger, Macromolecules, 23, 3520 (1990).

8. F. Heatley, Progress in NMR Spectroscopy, 13, 47 (1979).

9. F. Lauprêtre, C. Noël, and L. Monnerie, J. Polym. Sci., Polym. Phys. Ed., 15, 2127 (1977).

10. B. Chu, "Laser Light Scattering," 2nd ed., Academic, New York, 1991.

11. R. Pecora, Ed., "Dynamic Light Scattering," Plenum, New York, 1985.

12. A. T. Bullock, G. G. Cameron, and V. Krajewski, $J$. Phys. Chem., 80, 1792 (1976).

13. S. Mashimo, Macromolecules, 9, 91 (1976).
14. K. Adachi, Macromolecules, 23, 1816 (1990).

15. B. Valeur and L. Monnerie, J. Polym. Sci., Polym. Phys. Ed., 14, 11 (1976).

16. M. D. Ediger, Annu. Rev. Phys. Chem., 42, 225 (1991).

17. T. Sasaki and M. Yamamoto, Macromolecules, 22, 4009 (1989).

18. S. Yokotsuka, Y. Okada, Y. Tojo, T. Sasaki, and M. Yamamoto, Polym. J., 23, 95 (1991).

19. K. Adachi, Y. Imanishi, and T. Kotaka, J. Chem Soc., Faraday Trans. I, 85, 1065 (1989), 85, 1075 (1989), 85, 1083 (1989).

20. K. Adachi and T. Kotaka, Macromolecules, 18, 466 (1985).

21. K. Adachi, I. Nishi, H. Doi, and T. Kotaka, Macromolecules, 24, 5843 (1991).

22. D. Boese, F. Kremer, and L. J. Fetters, Macromolecules, 23, 829 (1990).

23. V. Veissier, J.-L. Viovy, and L. Monnerie, Polymer, 30, 1262 (1989).

24. D. A. Waldow, B. S. Johnson, P. D. Hyde, M. D. Ediger, T. Kitano, and K. Ito, Macromolecules, 22, 1345 (1989).

25. D. B. Adolf, M. D. Ediger, T. Kitano, and K. Ito, Macromolecules, 25, 867 (1992).

26. "Kagaku Binran Kiso Hen," 3rd ed., The Chemical Society of Japan, Ed., Maruzen, Tokyo, 1984, Chap. 6.

27. N. Hadjichristidis and J. E. L. Roovers, J. Polym. Sci., Polym. Phys. Ed., 12, 2521 (1974).

28. Ya. I. Poddubnyi and E. G. Ehrenburg, J Polym. Sci., 57, 545 (1962).

29. K. Ono, Y. Okada, S. Yokotsuka, S. Ito, and M. Yamamoto, Polym. J., 26, 199 (1994).

30. T. Sasaki, M. Yamamoto, and Y. Nishijima, Macromolecules, 21, 610 (1988).

31. C. K. Hall and E. Helfand, J. Chem. Phys., 77, 3275 (1982).

32. H. A. Kramers, Physica, 7, 284 (1940).

33. K. Ono, Y. Okada, S. Yokotsuka, T. Sasaki, and M. Yamamoto, Macromolecules, to be submitted.

34. P. J. Flory, "Principles of Polymer Chemistry," Cornell University Press, Ithaca, New York, 1953. 